\title{
A NETWORK OF INTELLIGENT PROXIMITY IOT DEVICES FOR OBJECT LOCALIZATION, INFORMATION COMMUNICATION, AND DATA ANALYTICS BASED ON CROWDSOURCING
}

\author{
Mike $\mathrm{Qu}^{1}$ and $\mathrm{Yu} \mathrm{Sun}^{2}$ \\ ${ }^{1}$ Northwood High School, Irvine, CA 92602 \\ ${ }^{2}$ California State Polytechnic University, Pomona, CA 91768
}

\begin{abstract}
While the advancements of technology have benefited the society in many ways, certain problems remain, and one such problem is the issue of lost people and pets. Current technology has offered many solutions to this problem, yet none is able to encompass all the core aspects to bring an end to the problem. My research proposes a solution that is practical, durable and reliable -- a proximity sensor device powered by a crowd of people, by using their mobile devices as receiving stations of the service, extensively increasing the effectiveness of this service in especially urban and suburban areas where there is a high population density.
\end{abstract}

\section{KEYWORDS}

Beacon, Device Network, Crowdsourcing, Double-blind, Artificial Intelligence.

\section{INTRODUCTION}

Many pet owners have once come across the trouble of losing their pets. While some animals are eventually retrieved by their owners, others are not so fortunate. In fact, 6.5 million companion animals enter U.S. animal shelters every year. Among them, only 710,000 are returned safely to their owners while more than 1.5 million are euthanized due to a lack of housing capacity in animal shelters [1].

To combat this growing problem, pet owners and vendors have developed a variety of services, including GPS location [2], microchips and written tags. While each type of service has their own merits, there are definitely shortcomings to them as well. GPS location is often expensive as it requires a variety of infrastructure support, including ground stations and positioning satellites to function properly. GPS signals are also very limited in indoor locations, underground areas, and places with large buildings and structures. Aside from that, GPS devices require charging on a regular due to their rapid consumption of energy, both posing a hassle to pet owners and significantly hindering the effectiveness of such a device. On the other hand, the implementation 
of microchips and written tags are a lot less costly, and that they are able to accurately identify a pet to animal control services makes these methods seem practical. However, when the fact that only 6.5 million of the approximately 70 million stray animals living are taken into animal shelters in the U.S. is taken into consideration, the aforementioned methods seem obsolete as there is no absolute guarantee that one's lost pet would eventually end up in one of the 3,500 animal shelters throughout the country.

Missing companion animals, a seemingly small problem is just the tip of an iceberg of a much more important issue. Many other groups in the population suffering from specific medical conditions, such as Alzheimer's and dementia face the same challenges in the society. According to the Alzheimer's association, six in ten patients would wander and oftentimes, they may not remember their home address, and can become disoriented even in familiar places [3]. Aside from that, lost children who are usually unaware of their surroundings also account for a large portion of this problem. According to the FBI, there were 464,324 [4] entries for missing children in 2017 , and by providing reliable and accurate locations of potential runaway children, this number can be greatly decreased.

A sprawling population in urban and suburban areas brings various social problems, including the increasing numbers of runaway people and pets call for a more accurate, reliable and practical approach to combat the problem. An increasing population for companion animals has overloaded the current system of animal shelters, and the growing population density also poses an increasing threat to particular groups, especially those who suffer from conditions that affect their capabilities of living independently.

A low-cost, reliable, and crowd-based technology using individuals' mobile devices as receiving stations to map and locate lost items used in accordance the iBeacon technology [5-7]. The iBeacon technology is able to emit Bluetooth 4.0 [15-18] signals and is based on physical proximity sensor devices known as beacons. These devices are small, durable and reliable. This particular technology requires no pairing, which means that mobile devices, such as cell phones and hardware devices could all receive the signal and prompt a specific function, from retrieving the satellite location of that device to sending a statement or message to that device. A number of such mobile devices in the same area, simultaneously sensing a beacon, could provide a wellpositioned and accurate satellite location for that purpose.

The rest of the paper is organized as follows: Section 2 details the challenges in this research project; one solution is presented in Section 3, followed by showing a discussion in Section 4; we compare the related works in Section 5, and Section 6 offers the conclusion remarks and future work directions.

\section{Challenges}

Due to the complexity of this solution, A few challenges are encountered during the development of this system.

\subsection{Challenge 1: Potential Lack of Coverage}

Without a dense network propagated from a number of devices located in a small area, the system would be not as effective because of the nature of this system, which requires a number of mobile 
devices to function. Furthermore, it calls for the density of mobile devices, instead of dispersity. Having multiple devices in a small and close-knit community could benefit its residents more than having the same number of devices across a much larger city. Therefore, we need to overcome the challenge by finding a solution to increase the coverage and make it as effective as other methods.

\subsection{Challenge 2: Lack of Appealing Features to the Public}

The ultimate usage of this device could be somehow limited as the ultimate beneficiaries are those who need this service the most, such as pet owners to track their pets, concerned family members to know the whereabouts of potential wandering patients, or parents who have young children who are unaware of their surroundings. Due to this, what this service has to offer could be slightly limited to those who do not require this service. Thus, we need to provide flexibility for users when they are using such devices.

\subsection{Challenge 3: Risk of Privacy}

Since any device could be used as a receiving station for a beacon, privacy could be an important issue in the development of this product. The fact that all beacon-owners could modify the database of information storage could have a negative effect on the practicality and reliability of this system. Therefore, it is necessary to solve the problem and avoid privacy issues in usage of such devices.

\section{METHODOLOGY}

\subsection{Overview of the Solution}

The underlying concept of this solution is the use of the powerful iBeacon technology on compact "beacons", which could emit a consistent Bluetooth 4.0 signal without the need to pair with a mobile device. When such a signal is received by either a hardware receiver or a mobile device, the device or receiver would log its own location, and publish that location in terms of longitude and latitude to a database, updating the location to indicate the approximate position of that beacon. This data can be downloaded from the database and displayed on the owner's device. Due to concerns for privacy, the users would have the option to turn on or turn off their abilities to give away their location to potential beacons in its range.

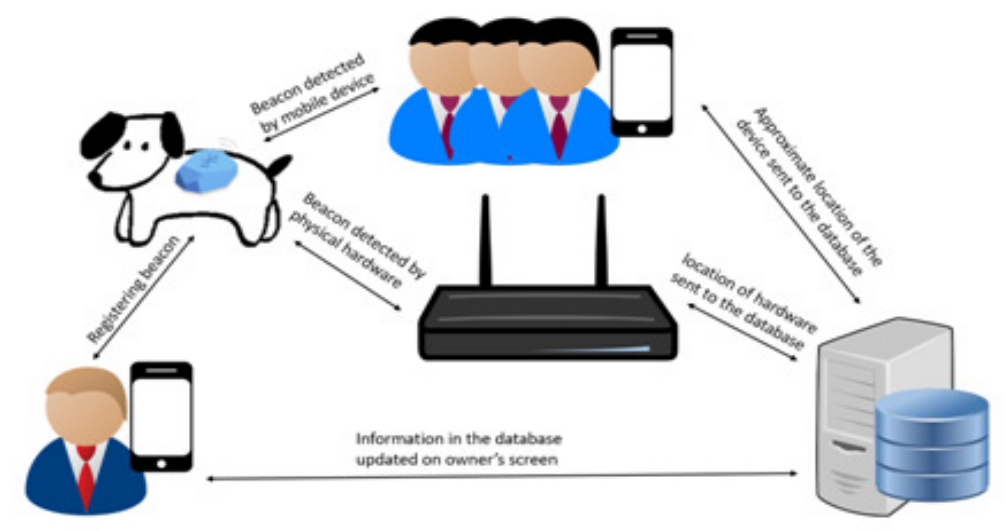

Figure 1. The overview of the solution 


\subsection{Crowd Analysis}

As mentioned above, this system functions best when used under a dense coverage of mobile devices. The more devices there are in one area, the more accurate the provided location will be. This situation can best be modelled by a Venn Diagram -- The three circles (in Figure 2) represent three different devices, either a physical signal receiver or a mobile device, that are of certain distances among one another. Having only one device is the least accurate, as the potential location of that beacon could be anywhere in the whole circle. Having two devices significantly improves the amount of error in locating that beacon, but its effectiveness could greatly vary. While having three or more devices could limit the beacon's location in a small, triangular area, which would be the most accurate.

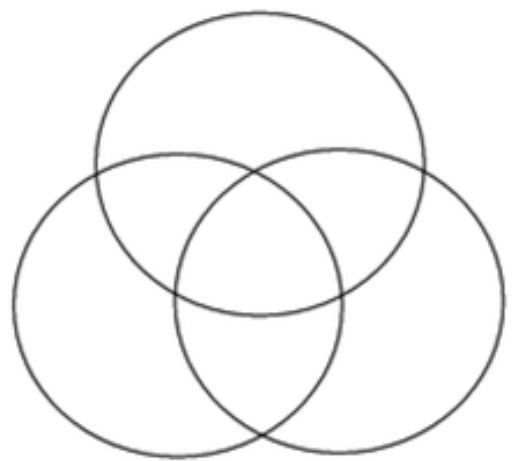

Figure 2. The range of three different devices

\subsection{Physical Capacities of a Beacon}

As mentioned above, Beacons are usually small, about an inch in diameter, and around a quarter of an inch in height. This attribute makes the beacons very light and durable. These beacons require coin batteries and require replacements once every one to two years, making them reliable under most circumstances. Beacons also vary in coverage area, ranging from around 20 meters to more than 350 meters for advanced models [4], but sensors could distinguish different strengths of iBeacon signal, which can be used in calculations to determine the approximate location of that beacon.

\subsection{Signal Receiver Hardware}

The signal receiver hardware is a mini-computer that requires both a power outlet and a Wi-Fi connection. This device is able to scan the surrounding area once every one tenth of a second for Bluetooth signals. Although this mini-computer is unable to locate itself due to the lack of a GPS location service, it usually stays stationary in at key locations, such as community gates, shopping centers, street intersections etc. to maximize how effective it is.

\subsection{Mobile Application}

The mobile application contains three screens: One for navigation, one for monitoring the registered beacons that belong to a user and one for initial registration of a beacon. It is able to pinpoint the exact location of the mobile device or the hardware signal receiver that last came across a signal from that iBeacon. There is also a drop-down menu that allows for one to track 
multiple beacons simultaneously, and each of them have a unique Beacon ID, a user-set device name and a password that can be established through registering the beacon. There is also a manual switch that allows one to enable or disable their device's ability to detect signals.

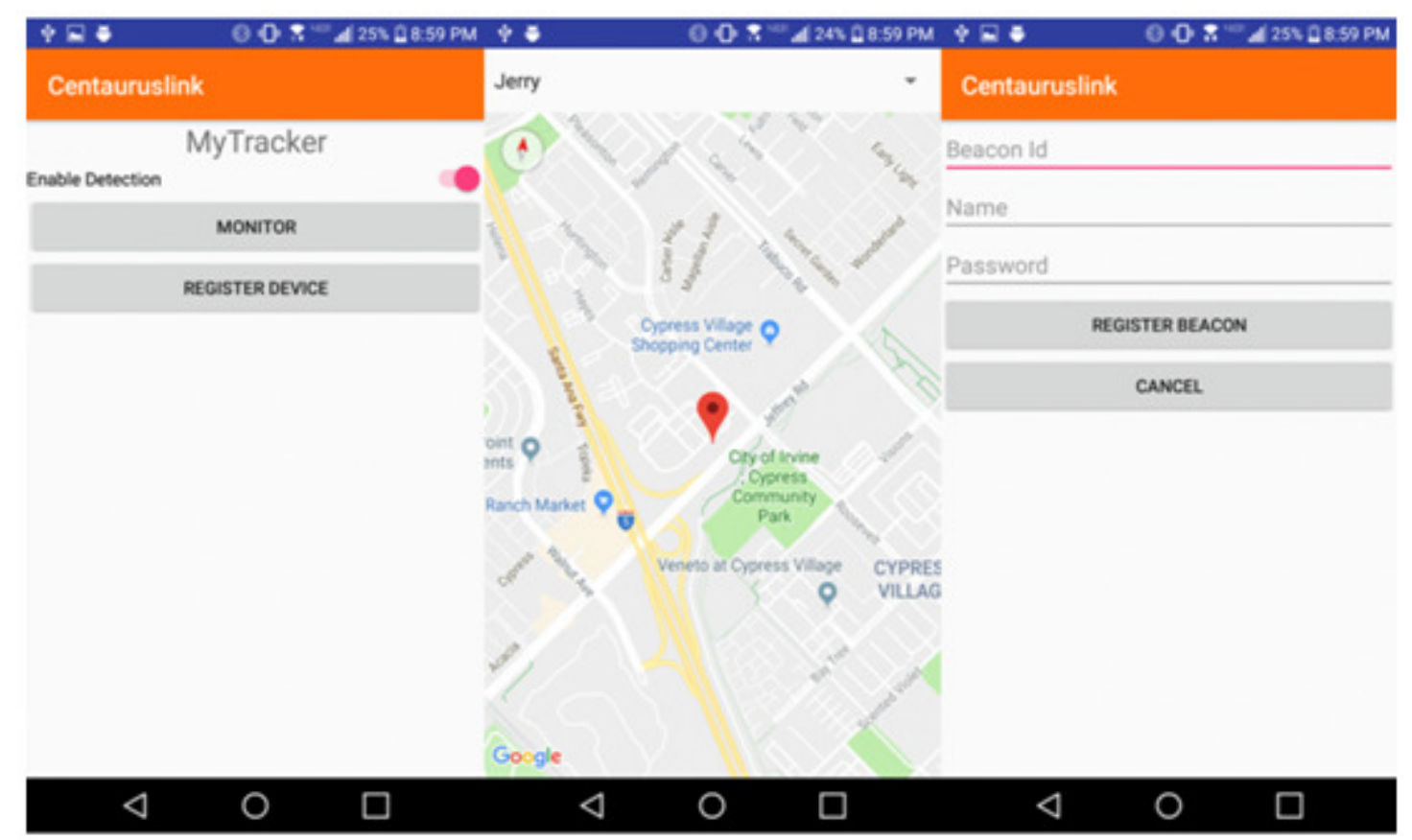

Figure 3. The Screenshots of the mobile application

\section{DISCUSSION}

To both increase the popularity of this service and improve signal coverage, a rewards mechanism can be put in this system. Since every mobile device that has the app installed could act as a sensor to any nearby beacon, anybody could contribute to locating service "accidentally" even if they are just moving along their daily routine. Everyone who contributes to the ultimate finding of a lost pet, for example, could receive some benefits issued by the pet's owner, such as financial rewards, or even words of gratitude. By incorporating this element of surprise to find something and potentially receive rewards even when one is minding their own business, the public can be encouraged to use this service regardless of whether this technology is of personal importance to them. To improve the security of this system, a sign-in mechanism could be implemented that incorporates registering a device, people would be limited to viewing their own beacons only. And receiving the location of a beacon would be a double-blind process. The owner wouldn't know who contributed to the location, and the locator wouldn't know whose beacon it is that is being located by his or her device. This both improves the security of the exchange of information and reinforces the element of surprise mentioned above.

\section{RELATED WORK}

iBeacon technology refers to a new generation of low-cost devices which is allowing marketers to track the exact location of consumers via their mobile devices [5-7]. BLE beacons [8] emit a signal that can be received by a BLE-enabled device within a close range. Apps can be built to 
cause events to be triggered within an instant of a device coming within the detectable range of the beacon. Moreover, the device can calculate how near or far away it is from the beacon, meaning that different events can be triggered depending on whether a device is within a range. A device can identify numerous beacons simultaneously and, by calculating its relative distance from each of the beacons, the device can gain an element of location awareness.

BLE beacons are important because they overcome challenges, such as secure, proximity-based communication, indoor geo-location, and wide-reaching distribution. It has a variety of application [9-14]. In this paper, we use iBeacon technology based on the technique of crowdsourcing. to solve the problem of lost pets, runaway children or wandering Alzheimer's patients, and similar needs in application.

\section{CONCLUSION AND FUTURE WORK}

In this project, we proposed a this system is designed to solve the problem of lost pets, runaway children or wandering Alzheimer's patients as well as anyone else who are struggling in a similar condition. By using mobile devices as receiving stations for signals in addition to having physical and designated receivers, the coverage of this service can be greatly improved to benefit the society and people as a whole.

This project also has the potential to become something much more sophisticated. Using such a proximity sensor device, one could request information based on their proximity with a beacon. This specific implementation could be used in areas such as museums for guided tours, showrooms for pricing and item specifics and billboards for further information. Furthermore, analysis could also be done on collected data. For example, one could determine what is a popular amusement park ride, how many customers did a department store have in one hour and so on. The future to this technology is endless and it is important to make it so that it can best benefit our society as a whole.

As for the future work, we will investigate accuracy and efficiency of the system. We also would like to explore the possibility of applying the system on other domains and providing convenience for human being.

\section{REFERENCES}

[1] Scarborough, Pet al, Prachi Bhatnagar, Kremlin Wickramasinghe, Kate Smolina, Colin Mitchell, and M. Rayner. "Coronary heart disease statistics 2010 edition." British Health Foundation Health Promotion research group, Department of Public Health, University of Oxford (2010).

[2] Bajaj, Rashmi, Samantha Lalinda Ranaweera, and Dharma P. Agrawal. "GPS: location-tracking technology." Computer 4 (2002): 92-94.

[3] Glenner, George G., and Caine W. Wong. "Alzheimer's disease: initial report of the purification and characterization of a novel cerebrovascular amyloid protein." Biochemical and biophysical research communications 120, no. 3 (1984): 885-890.

[4] Person, NCIC Missing. "Unidentified Person Statistics for 2009." National Crime Information Center, Federal Bureau of Investigation (2014). 
[5] Newman, Nic. "Apple iBeacon technology briefing." Journal of Direct, Data and Digital Marketing Practice 15, no. 3 (2014): 222-225.

[6] Koühne, Markus, and Jürgen Sieck. "Location-based services with iBeacon technology." In Artificial Intelligence, Modelling and Simulation (AIMS), 2014 2nd International Conference on, pp. 315-321. IEEE, 2014.

[7] Lin, Xin-Yu, Te-Wei Ho, Cheng-Chung Fang, Zui-Shen Yen, Bey-Jing Yang, and Feipei Lai. "A mobile indoor positioning system based on iBeacon technology." In Engineering in Medicine and Biology Society (EMBC), 2015 37th Annual International Conference of the IEEE, pp. 4970-4973. IEEE, 2015.

[8] Ji, Myungin, Jooyoung Kim, Juil Jeon, and Youngsu Cho. "Analysis of positioning accuracy corresponding to the number of BLE beacons in indoor positioning system." In Advanced Communication Technology (ICACT), 2015 17th International Conference on, pp. 92-95. IEEE, 2015.

[9] Yang, Jingjing, Zhihui Wang, and Xiao Zhang. "An ibeacon-based indoor positioning systems for hospitals." International Journal of Smart Home 9, no. 7 (2015): 161-168.

[10] Corna, Andrea, L. Fontana, A. A. Nacci, and Donatella Sciuto. "Occupancy detection via iBeacon on Android devices for smart building management." In Proceedings of the 2015 Design, Automation \& Test in Europe Conference \& Exhibition, pp. 629-632. EDA Consortium, 2015.

[11] Burzacca, P., M. Mircoli, S. Mitolo, and A. Polzonetti. "“iBeacon" technology that will make possible Internet of Things." (2014): 159-165.

[12] Conte, Giorgio, Massimo De Marchi, Alessandro Antonio Nacci, Vincenzo Rana, and Donatella Sciuto. "BlueSentinel: a first approach using iBeacon for an energy efficient occupancy detection system." In BuildSys@ SenSys, pp. 11-19. 2014.

[13] Akinsiku, Adegboyega, and Divyesh Jadav. "BeaSmart: A beacon enabled smarter workplace." In Network Operations and Management Symposium (NOMS), 2016 IEEE/IFIP, pp. 1269-1272. IEEE, 2016.

[14] Fard, Hadis Kakanejadi, Yuanzhu Chen, and Kyung Kook Son. "Indoor positioning of mobile devices with agile iBeacon deployment." In Electrical and Computer Engineering (CCECE), 2015 IEEE 28th Canadian Conference on, pp. 275-279. IEEE, 2015.

[15] Bhagwat, Pravin. "Bluetooth: technology for short-range wireless apps." IEEE Internet Computing 5, no. 3 (2001): 96-103.

[16] McCurdy, Roger A. "Emergency assistance system using bluetooth technology." U.S. Patent 6,340,928, issued January 22, 2002.

[17] Erasala, Naveen, and David C. Yen. "Bluetooth technology: a strategic analysis of its role in global 3G wireless communication era." Computer Standards \& Interfaces 24, no. 3 (2002): 193-206.

[18] Tajika, Yosuke, Takeshi Saito, Keiichi Teramoto, Naohisa Oosaka, and Masao Isshiki. "Networked home appliance system using Bluetooth technology integrating appliance control/monitoring with Internet service." IEEE Transactions on Consumer Electronics 49, no. 4 (2003): 1043-1048. 